\title{
Filigrane
}

Écoutes psychanalytiques

\section{L'apport de la psychanalyse dans les prises en charges hospitalières d'adolescents : l'expérience du département de psychiatrie de l'adolescent et du jeune adulte de l'Institut Mutualiste Montsouris (1963-2015)}

\section{Guillemette Balsan}

Volume 24, numéro 2, automne 2015

Le devenir de la psychanalyse. Que nous disent les pratiques institutionnelles?

URI : https://id.erudit.org/iderudit/1036530ar

DOI : https://doi.org/10.7202/1036530ar

Aller au sommaire du numéro

Éditeur(s)

Revue Santé mentale au Québec

ISSN

1192-1412 (imprimé)

1911-4656 (numérique)

Découvrir la revue

Citer cet article

Balsan, G. (2015). L’apport de la psychanalyse dans les prises en charges hospitalières d'adolescents : l'expérience du département de psychiatrie de

l'adolescent et du jeune adulte de l'Institut Mutualiste Montsouris (1963-2015).

Filigrane, 24(2), 41-56. https://doi.org/10.7202/1036530ar
Résumé de l'article

Retracer l'histoire de l'institution permet de mettre en exergue les diverses strates de ce qui fonde aujourd'hui les soins psychiatriques auprès des adolescents à l'Institut Mutualiste Montsouris (IMM). Est alors interrogé ce qu'il en est à ce jour de la transmission de cet héritage, et en particulier de la place de la psychanalyse. L'IMM est organisé en différentes unités de soins articulées de façon transversale. Ce dispositif s’appuie sur la plurifocalité et la multidisciplinarité, au regard des spécificités de la psychopathologie de l'adolescent, notamment les caractéristiques transférentielles afférentes à la problématique centrale de la séparation-autonomisation-subjectivation. 


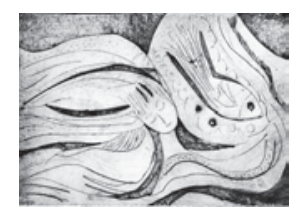

L'apport de la psychanalyse dans les prises en charges hospitalières d'adolescents: l'expérience du département de psychiatrie de I'adolescent et du jeune
adulte de I'Institut Mutualiste
Montsouris (1963-2015)

\author{
Guillemette Balsan
}

Retracer l'histoire de l'institution permet de mettre en exergue les diverses strates de ce qui fonde aujourd'hui les soins psychiatriques auprès des adolescents à l'Institut Mutualiste Montsouris (IMM). Est alors interrogé ce qu'il en est à ce jour de la transmission de cet héritage, et en particulier de la place de la psychanalyse. L'IMM est organisé en différentes unités de soins articulées de façon transversale. Ce dispositif s'appuie sur la plurifocalité et la multidisciplinarité, au regard des spécificités de la psychopathologie de l'adolescent, notamment les caractéristiques transférentielles afférentes à la problématique centrale de la séparation-autonomisation-subjectivation.

La maladie n'est que le modèle choisi en face d'un patient et le malade n'est choisi qu'en raison des moyens d'action qui sont à la disposition des auteurs et des hypothèses pathologiques qu'ils bâtissent.

Georges Daumezon

\title{
II était une fois... I'Institut Mutualiste Montsouris (IMM)
}

e lieu d'où nous parlons est celui d'une expérience clinique institutionnelle auprès d'adolescents déjà "vieille» d'un demi-siècle. Cinquante ans: c'est le milieu de vie critique où l'on interroge ce que l'on a reçu en héritage, ce que l'on en a fait, et son devenir.

Questionner l'histoire, c'est s'intéresser aux traditions et à leur transmission — comment passer le témoin, la flamme — esprit et élan vital? 
Évoquer la tradition, c'est peut-être prendre le risque d'être «has been » sur fond de nostalgie (hélas, ils nous quittent les uns après les autres: Serge Lebovici en 2000, Pierre Fédida en 2002, André Green et Michel Soulé en 2012, Jean-Bertrand Pontalis et Roger Misès en 2013, Jean Gillibert en 2014), ou encore de passer pour «réac» quand nous prônons la valeur maturative et thérapeutique de la séparation, des règles et des contraintes - ni télé, ni tablettes, encore moins de téléphone et d'internet; pas de menus à la carte... Tout n'est pas permis: il n'est pas «interdit d'interdire ${ }^{1} »$ ! Comme par exemple les relations amoureuses entre patients.

Et de l'importance de la famille, de la différence des sexes et des générations; une conception des limites comme condition du rêver, sous-tendue par les notions d'étayage et d'environnement rassurant, selon une acception qui pourrait appartenir à l'éthologie, nécessaire - mais non suffisante - à la naissance des autoérotismes et de la vie fantasmatique.

La transmission pourrait être appréhendée selon un double axe, vertical filiatif, et horizontal affiliatif.

Une histoire? C'est ce qui à la fois véhicule une tradition, en est la mémoire, et est l'outil, l'instrument, vecteur de la transmission. Cette histoire se construit à partir des traces, des empreintes laissées par les différentes générations de psychiatres et de soignants.

Ainsi, trois générations se sont succédé depuis la création en 1963 du département de psychiatrie pour adolescents, chacune façonnant l'institution selon son orientation théorique, ses espoirs et ses croyances.

\section{L'esprit de famille "Flavigny »: origine et mythes}

Les premières pierres de l'édifice furent posées par le $\mathrm{P}^{\mathrm{r}}$ Hubert Flavigny, psychiatre humaniste et chrétien de gauche. La légende raconte que chez lui et son épouse Maité, il y avait toujours un lit et un couvert pour celui qui se présenterait. Sa maison demeurait ouverte aux jeunes délinquants en errance, hébergés là plusieurs jours ou plusieurs mois; «SDF» d'hier, et ancêtre des foyers d'urgence, du $115^{2}$, ou des Centres d'Accueil et de Crise ${ }^{3}$ (CAC)? Autre réminiscence que nous confie avec plaisir B., aujourd'hui retraité, et qui connut le $\mathrm{P}^{\mathrm{r}}$ Flavigny alors qu'il était interne: le souvenir d'une partie de football avec son Maître en shorts courant derrière le ballon! Un second médecin se rappelle combien ce chef de service «œcuménique» mettait un point d'honneur à composer son équipe de soignants d'horizons très éloignés, qu'il réunissait autour d'un méchoui ou à l'occasion d'autres festivités pour créer du lien entre eux. De fait, Hubert Flavigny était connu 
pour avoir un remarquable sens de la fête. Vie privée et engagement professionnel étaient indissociables, comme pour beaucoup de psychiatres d'après-guerre, dans la mouvance rebelle des lieux comme Saint-Alban avec François Tosquelles, ou la clinique de La Borde avec Jean Oury (qui avait été interne chez le premier), issue de l'esprit de la résistance qui avait uni des hommes souvent très différents dans leur lutte pour la liberté.

Hubert Flavigny référait ainsi le soin psychiatrique au champ psychosocial et affirmait la nécessité d'une fraternité des contraires et sa foi en la thérapie institutionnelle née après 1945. Les soignants, à son invite, abandonnèrent leurs blouses blanches; les malades participaient aux prises de décision concernant le quotidien de l'institution, au même titre que toute personne y travaillant (cuisinier, personnel d'entretien, jardinier...) considérée comme autant de cliniciens. C'est ainsi que le $\mathrm{P}^{\mathrm{r}}$ Flavigny favorisa l'embauche de personnes sans diplôme, ni même une expérience de soignant mais en qui il décelait une qualité relationnelle particulière (qu'il qualifiait de «naïve») et une habilité technique (comme la peinture, la menuiserie, le tricot etc.; activités manuelles précurseures des médiations thérapeutiques), inventant pour eux le statut de "moniteur soignant». Ainsi, avec H. Flavigny, le soin devient avant tout ouverture vers l'autre, accordant valeur au temps et au lieu de l'accueil. Être ensemble relève de la fonction soignante de l'expérience vécue. Expérimenter des rencontres nouvelles, le «créer» ensemble, partage d'instants et d'affects.

Cette brève évocation souligne l'importance de la dimension groupale dans le soin, avec la représentation peut-être aujourd'hui idéalisée d'une équipe disparate faisant corps autour d'un «chef» charismatique. (N'est-ce pas comme cela que naissent les mythes?).

Aussi, nous nous représentons la jubilation des jeunes internes d'alors (qui sont ou furent nos séniors) courant d'un séminaire à l'autre; la formation au psychodrame aux côtés d'Évelyne Kestemberg; l'ASM13 ${ }^{4} \ldots$ le premier secteur psychiatrique en France, expérience «pilote» et illustration vivante de la psychothérapie institutionnelle faisant référence à la psychanalyse, pas tant comme dispositif de soin (celui de la cure analytique classique de l'adulte) mais plutôt comme modèle de compréhension de la pathologie mentale.

Avec cette seconde génération, que nous avons baptisée du nom de l'aîné, le $\mathrm{P}^{\mathrm{r}}$ Philippe Jeammet, le service de Montsouris prend un cours psychodynamique qui s'inscrit dans la démarche de nombre de psychiatres et d'hôpitaux psychiatriques de pratiquer une "psychanalyse sans divan» à 
l'appel de Paul-Claude Racamier (Racamier, 2008), René Diatkine (un des trois auteurs du $L S D^{5}$ avec Serge Lebovici et Michel Soulé, qui est toujours aujourd'hui un ouvrage de référence en pédopsychiatrie), Philippe Paumelle et tant d'autres.

\section{La génération "Philippe Jeammet »: des psychanalystes à I'hôpital ${ }^{6}$... et des réanimateurs en psychiatrie}

Cette génération questionne l'apport de la psychanalyse à la psychiatrie (Jeammet, 1996) avec une réflexion sur les aménagements du cadre psychothérapeutique de la cure analytique auprès de l'adolescent, du dispositif individuel aux groupes (Jeammet, 2002). L'on retrouve la créativité du trépied psychanalytique qui associe une pratique clinique, une théorisation toujours en cours, et un mode de connaissance des processus psychiques. Philippe Jeammet a développé un intérêt particulier pour la prise en charge des troubles des conduites alimentaires (TCA) à l'adolescence (Jeammet, 1989), lesquels ont valeur de paradigme pour la compréhension de la psychopathologie de l'adolescent. Cette clinique centrée sur le corps, et le modèle théorico-clinique qui en découle, mettent ainsi en exergue la problématique de séparation-autonomisation/dépendance commune aux troubles psychiques émergeant à cet âge charnière, entre l'enfance et l'âge adulte, initié par la puberté (Azoulay et Chabert, 1998).

À partir des années 2000, Philippe Jeammet, tout en conservant un cadre psychodynamique et un dialogue avec les psychanalystes dans le service et "hors les murs", adopte une approche davantage inspirée par les travaux des éthologues et de la théorie de l'attachement (de J. Bowlby à P. Fonagy), ce qui redouble l'intérêt qu'il avait très tôt porté à la prise en compte de l'environnement (la réalité externe) tant pour l'évaluation clinique que pour la thérapeutique. En effet, $\mathrm{P}$. Jeammet avait le souci de travailler en particulier avec les familles, mais aussi d'intégrer des soins somatiques aux soins psychiatriques, avec le concours notamment du service de médecine interne, ainsi qu'en étroite collaboration avec les réanimateurs de l'IMM pour la prise en charge de patients anorexiques cachectiques (Tric, Agman, Godart, Benmansour et Lamer, 2004).

\section{L'équipe de Maurice Corcos ou "la horde des frères " : du " chaos " et de la ré-animation/création somato-psychique}

La troisième génération, à la suite du $\mathrm{P}^{\mathrm{r}}$ Maurice Corcos, donne un nouveau souffle à l'approche psychanalytique dans les soins avec une prise en 
compte renouvelée de la dynamique pulsionnelle à l'œuvre. D'où l'importance du conflit considéré comme signe de bonne santé de l'institution. De la valeur du chaos au sens de l'hybris de l'Antiquité grecque, mais aussi selon la définition qu'en donne D.W. Winnicott dans La nature humaine (Winnicott, 1954). Désorganisation, vacillement identitaire, déliaisons qui permettent de nouvelles liaisons, potentiel de remaniement, ferment du changement lors des métamorphoses du corps adolescent.

Les frères? Disputations chères au $\mathrm{P}^{\mathrm{r}}$ Corcos où joutent les tenants des théories de l'attachement, les psychanalystes, les comportementalistes, et les adeptes de la remédiation cognitive, de la régulation émotionnelle ou encore de l'éducation thérapeutique; les obsessionnels et les hystériques, les angoissés et les dépressifs... tous avec une pointe de sensitivité qui traduit peutêtre l'intensité de l'implication de chaque soignant dans la vie institutionnelle, son engagement avec ses écueils comme ses gratifications narcissiques.

L'approche théorico-clinique de Maurice Corcos dessine des perspectives novatrices aux modèles psychosomatiques en psychanalyse (Carton, Chabert et Corcos, 2011; Corcos, 2010, 2011). La primauté de la sensorialité, de la pulsionnalisation de cette dernière qui donne forme au corps érogène et matière aux autoérotismes, est au cœur d'une réflexion qui cherche des voies nouvelles d'une possible réanimation psychique: réanimation des autoérotismes pour consolider, panser les fragilités narcissiques de l'adolescent afin que ce dernier prenne le risque de s'ouvrir au monde sans se sentir menacé dans son identité.

Les soins sont accordés à une compréhension génétique des symptômes: le recours au corps, les attaques du corps sur un mode addictif à l'adolescence, dans l'après-coup des heurts et malheurs des interactions précoces mère-bébé, notamment des vécus d'éprouvés anaclitiques (au mieux) comme négatifs d'une discontinuité de la présence maternelle (notion de «défaillance du maternel»). La reviviscence des éprouvés de lâchage, de néantisation à l'adolescence fait écho trop souvent à une dépression maternelle périnatale et à une histoire familiale transgénérationnelle organisée autour d'un noyau mélancolique (masqué par des conduites addictives dont les TCA).

Confrontant processus thérapeutiques et processus de création, Maurice Corcos appelle à une poïétique et à une esthétique de la rencontre dans le soin; une éthique de la relation soignante sur le modèle de l'engagement «amoureux» (Lucien Israël) : le lieu d'utopie (Baruch Spinoza), lieu de créativité qui est mouvement/geste psychothérapeutique. 


\section{Quelle place donner aujourd'hui à la psychanalyse? Le dispositif de soin actuel: de nouvelles orientations sur fond de continuité}

Quelle place pour la psychanalyse aujourd'hui en psychiatrie de l'adolescent et du jeune adulte? Cette question exprimerait-elle une tentative de résistance et de survie ou est-elle une interpellation militante? Le service de Montsouris serait-il un des derniers bastions de la psychothérapie institutionnelle en pédopsychiatrie, ou bien une figure d'avant-garde?

Autrement dit, cette pratique du soin est-elle encore possible? Cette interrogation teintée de nostalgie traduirait l'idée d'un quelque chose en voie de disparition, d'extinction inéluctable, et que l'on tenterait ainsi de retenir, en vain, à regret. Elle révélerait peut-être aussi le refus du changement immanent à l'écoulement du temps qui nous échappe - oracle de mauvais augure annonçant la fin d'un monde. L'on traque alors les rescapés, les derniers témoins, les «Don Quichotte» de la thérapie institutionnelle.

Ces propos questionnent alors la place des mythes, la fonction des processus d'idéalisation et de désidéalisation, la force et l'intensité de l'éphémère; sujets d'actualité brûlante au temps adolescent.

Quel est donc l'enjeu? Maintenir vivante et dialectique la pluralité dans les soins, versus une tendance à une opérationnalisation (ou «manuellisation») de traitements efficaces mais froids, mécaniques et sans affect. Manuel diagnostique et statistique des troubles mentaux (DSM), programmes de régulation, adaptation, éducation, gestion... certification, cotations $\mathrm{T}_{2} \mathrm{~A}^{7}$ et RIM-P ${ }^{8}$ : tous, vocables d'une redoutable efficacité en termes de politique de coût (et encore, cela serait à vérifier), mais d'une radicale insignifiance face au patient.

De fait, c'est une question d'éthique. Une problématique de fond: celle de la singularité de la rencontre au cour du soin. D'où la nécessité d'une lutte constante contre la tentation d'un prêt-à-porter sanitaire, des protocoles et autres stratégies thérapeutiques, arsenal bien huilé, se référant à une pensée unique qui rassure face à la "peur du fou», de la folie, de l'irrationnel, l'indescriptible, l'irreprésentable: mécanisme de rejet de ce qui dérange, l'étranger, l'étrange, l'Umheimlich si proche du familier (dont le sens est compris dans le terme allemand) soit les émergences, l'irruption du pulsionnel.

Devrions-nous nous inquiéter du risque de dérive d'une rationalisation des démarches diagnostiques et thérapeutiques dont la fixité, la rigidité, annoncerait une logique totalitaire, ou une régression aux «temps» de la 
psychiatrie asilaire? La moyenne statistique n'a rarement satisfait le bonheur de chacun.

L'approche psychanalytique est à la fois essentielle et complémentaire (pour ne pas verser par excès dans le "tout psychique»), alors que le psychisme est par définition corporel (Freud, 1923). L’appréhension psychanalytique du symptôme, c'est non pas ignorer ce dernier, mais interroger sa fonction dans l'organisation psychique globale du jeune patient, ainsi que dans ses interactions avec son environnement (en l'occurrence la famille, le groupe de pairs, et par déplacement l'institution de soins, les soignants); c'est aussi, fidèle à Hippocrate, donner sens avec le patient à sa maladie en la resituant dans le cours de son histoire personnelle et familiale - une façon de se l'approprier. La psychanalyse propose ainsi un décentrement par rapport au symptôme dans la relation avec le thérapeute - support de réflexion (réflexivité et reflet). C'est une clinique du transfert, d'une rencontre, de rencontres qui, indicibles, se vivent dans l'affect, le surgissement de l'inouï, dans la langue des rêves; d'un impalpable entre les corps, la chair du fantasme, fulgurante figuration de l'inconnu (inconscient) qui nous meut dans son aura d'«inquiétante étrangeté».

L'étrangèreté d'être, ou encore le sentiment d'étrangeté (si perturbant, effet de discontinuité sur fond de continuité), ne serait-ce à quoi nous convoquerait tout particulièrement l'adolescence? Révélation/ouverture sous le prisme des métamorphoses pubertaires aux potentialités du moicorps, terreau/terrain du vacillement de soi - intermittences de l'être, évanouissement de l'âme (J. Rivière, correspondance avec A. Artaud, 1924), ou encore expérience de chaos winnicottien, source de créativité et d'inventivité.

Osons nous faire le porte-parole d'un engagement enthousiaste (qui ne saurait ignorer l'optimisme du pessimiste) : une profession de foi en une thérapeutique créative se fondant sur l'expérience institutionnelle selon la tradition vivante et originale de l'IMM, dans la filiation de Philippe Jeammet et de Maurice Corcos. Jacques Hochmann écrit dans le préambule à La consolation (Hochmann, 1994), qu'il était d'abord «entré en psychiatrie», puis qu'il «entrait en analyse». Comme d'autres entrent au séminaire, ou s'adonnent à l'étude du talmud. Une fois entré, peut-on en sortir? J'interroge ici le risque de dérive sectaire de tout engouement - enrôlement, si nécessaire peut-être soit-il. Au fond, de quel engagement s'agit-il? Qu'engage-t-on ainsi? L'on s'engage dans l'armée, dans un parti, un groupe. En anglais to be engaged signifie se fiancer, être lié/affilié, faire alliance. S'engager corps et âme, être 
fidèle à l'esprit de famille, défendre l'honneur du clan aussi, avec le risque de clôture sur soi et de sclérose.

Il y a la portée également d'un mouvement (aller vers, en; qui implique le corps, l'éprouvé d'affect), qui amorce, comme l'esquisse d'un arc-tension entre soi et l'autre, tension narcissico-objectale mise en corrélation avec l'articulation entre réalité interne et réalité externe, tel que nous y invite Philippe Jeammet dans un article de 1980 paru dans la Revue française de psychanalyse. Ainsi, l'engagement, c'est aussi l'expression d'une position assumée, celle de la référence à la psychanalyse dans le travail institutionnel en psychiatrie avec les adolescents. Ce texte demeure aujourd'hui une référence dans le champ de la psychopathologie de l'adolescent. L'auteur prend pour point de départ de sa réflexion sa pratique psychiatrique, en tant qu'à la fois psychanalyste d'adulte et pédopsychiatre. Il oriente cette clinique dans une perspective analytique: à savoir que la relation transférentielle est considérée comme outil d'évaluation diagnostique et moteur du traitement. Il s'inscrit ainsi dans une tradition de psychanalystes et de psychiatres qui cherchent à définir les spécificités de la prise en charge des adolescents, par comparaison à l'orthodoxie du cadre strict de la cure classique avec les adultes névrosés. Philippe Jeammet rend ainsi hommage au legs de Peter Blos, Moses et Eglé Laufer, Evelyne Kestemberg, Pierre Mâle...

L'attention du clinicien est particulièrement attirée par la massivité de l'investissement transférentiel chez l'adolescent, à l'origine de contreinvestissements tout aussi intenses, lesquels se manifestent souvent par de l'ennui, une neutralisation voire une rupture du traitement et des passages à l'acte. Ces acting out du jeune patient appellent alors à de nécessaires aménagements du cadre ou acting in du thérapeute, comme la verbalisation du contre-transfert, le rappel des règles et des limites, le travail avec les parents. L'acting in? Ce serait peut-être aussi cet engagement, cette implication du thérapeute dont je parlais plus haut, qui s'accorde à la dimension de l'agir propre à l'adolescence, où le recours à la réalité externe, le langage du corps, sont tentatives de maîtrise, de contrôle par la projection sur des supports externes d'un monde interne devenu chaos, bouleversé par la poussée pubertaire.

L'adolescence, par un double effet d'après-coup, révèle les carences infantiles (la vulnérabilité du monde interne) qui entrent en collusion avec la faillite de l'environnement externe. Cette alchimie rend alors d'autant plus difficile le processus de "seconde séparation» (ou de subjectivation selon Raymond Cahn). Ainsi, de façon générale, les troubles psychiques à 
l'adolescence relèvent d'une problématique de dépendance avec des aménagements défensifs propres à chaque patient. Ce dernier va être d'autant plus tributaire des objets externes que son monde interne (ses assises narcissiques) sera fragile; tout lien à l'autre va être vécu comme menaçant (avec le risque d'hémorragie narcissique) à la mesure du besoin qu'il en a (c'est-àdire de son degré de dépendance).

Le recours à la réalité externe, dont les conduites pathologiques, a fonction de contre-investissement d'une réalité interne défaillante. La compréhension économique et dynamique des troubles externalisés de l'adolescent implique alors que l'on prenne en considération la réalité externe, c'està-dire l'environnement immédiat du jeune, sa famille, les parents, le groupe de pairs, le cadre scolaire...

L'élaboration du dispositif de soins est issue de la conception que nous avons des troubles psychiques à l'adolescence et est le fruit d'une cocontruction de chaque instant: entre-deux/antre sur le modèle de l'espace transitionnel de D. W. Winnicott, le cadre des soins n'est jamais établi une fois pour toutes - ce qui le réduirait à être vide et sans vie —, il est « objet malléable» du fait même que les limites sont explicites (les règles sont faites pour être transgressées, répétait le $\mathrm{P}^{\mathrm{r}}$ Flavigny, pourtant connu pour être très pointilleux sur le cadre). Déplacements, vibrations, provocations qui infèrent au cadre du mouvement, source et conséquence de changement (de part et d'autre: tant du côté du soignant que du soigné).

Avant que d'interpréter, il faut pouvoir accueillir l'informe: un espacetemps pour recevoir, contenir ce matériau, dans ses allers et retours, au rythme oscillant de présence en évanescence. La condition de cette réceptivité résiderait en une élaboration primaire, au sens d'une digestion (Bion, 1983) qui, si elle n'est exprimée directement à l'adolescent, est le fondement du travail institutionnel: échanger entre soignants, parler, souffler, se lâcher; méthode qui accorde une place primordiale aux «mini staffs» pluriquotidiens, aux synthèses cliniques hebdomadaires autour des situations complexes, aux transmissions des internes après la garde tous les matins, aux «débriefings» après les groupes thérapeutiques (psychodrame, activités à médiations artistiques et corporelles), aux dites «supervisions» (des internes, ou des soignants impliqués dans une relation de soins duelle le plus souvent médiatisée - massage, socio-esthétique)... Il y a toujours un deuxième temps, second espace-temps emboîté dans le premier, qui nous invite à faire un pas de côté, ensemble. Cela suppose de savoir s'écouter les uns les autres, dans le respect de la parole de chacun; cela implique aussi de 
se décaler par rapport à soi. Se sentir la légitimité alors de s'exprimer, quels que soient la pensée, l'émotion, le geste qui viennent en association à la relation éprouvée avec le patient durant la journée.

Le département de psychiatrie de l'adolescent de l'IMM est organisé en différentes unités avec leurs spécificités invitant à penser l'articulation et le passage de l'une à l'autre.

Le principe de transversalité institutionnelle tend à une fluidité et à une souplesse de fonctionnement permettant d'accueillir le patient. Le cadre de soins, aux qualités de contenance et de pare-excitation, offre la possibilité au jeune de donner forme à l'informe (travail de figuration) par le truchement du jeu-ajustement des investissements relationnels, contournant l'écueil d'une structure trop rigide, laquelle mènerait à des impasses thérapeutiques, à la rupture d'avec les soins et, souvent, à la multiplication des passages à l'acte.

Sont ainsi regroupés depuis le déménagement en 1996 dans le bâtiment actuel, qui venait alors tout juste d'être érigé (les données entre parenthèses sont indicatives de l'activité clinique de 2014):

Au rez-de-chaussée:

— L'unité de jour (4251 journées/74 patients/96 séjours) accueille les adolescents durant la journée; les soins sont individualisés allant de la prescription d'une activité thérapeutique à un emploi du temps structuré sur cinq jours de présence hebdomadaire. (Azoulay et Chabert, 1998).

- L'unité de recherche clinique.

Au premier étage:

- Le centre de consultation Hubert Flavigny (file active de 912 patients) propose des consultations d'évaluation clinique à des adolescents âgés de 13 à 18 ans avec un psychiatre ou un psychologue, afin de déterminer au mieux avec le jeune et ses parents le projet thérapeutique et le cadre de la prise en charge (thérapie individuelle et/ou familiale, prise en charge bifocale, hospitalisation, aménagement de la scolarité, orientation vers une clinique soins-études, prescription d'un traitement psychotrope, collaboration avec le médecin traitant — souvent le pédiatre ou le médecin généraliste).

- L'unité d'hospitalisation de crise (2305 jours/90 patients/114 séjours; taux d'occupation: $76 \%$ ) peut accueillir huit adolescents dans des contextes de crise, c'est-à-dire des moments aigus, critiques, révélateurs de conflits ou de tensions intrapsychiques et/ou intrafamiliaux pour des séjours d'une durée en général inférieure à un mois. 
Au second et dernier étage:

- L'unité d'hospitalisation de moyenne à longue durée (8322 jours/83 patients/149 séjours; taux d'occupation: $79 \%$ ) accueille trente adolescents pour une durée le plus souvent de plusieurs mois et dont plus des deux tiers souffrent d'anorexie sévère (avec une majorité de filles) nécessitant une prise en charge spécifique associant soins psychiatriques et nutritionnels.

Pour mémoire, le cadre de soins est conçu en lien avec un modèle original psychodynamique de compréhension de la psychopathologie de l'adolescence. Celui-ci s'articule à la problématique centrale de la séparation inhérente au mouvement d'individuation-subjectivation, et réactualisée à l'adolescence. En effet, la proximité et l'intensité de la fantasmatique inconsciente œedipienne, incestuelle et parricide, génèrent une hypersexualisation des liens, qui nécessite un aménagement de la distance d'avec les figures parentales. Tout ce qui renvoie alors à un état de dépendance vis-à-vis de ces imagos devient insupportable.

L'usage du terme de psychodynamique, préféré à celui de psychanalytique, met l'accent sur la pulsion, sur la dynamique de la dialectique entre le latent et le manifeste, mais aussi sur la fonction défensive du symptôme dans une tentative de contenir l'excitation pulsionnelle devenue explosive avec la puberté. Ce débordement tend à prendre forme-exutoire dans l'agir, mode d'expression prépondérant chez le jeune adolescent. L'action manifeste inversant le vécu de soumission, de réception passive des métamorphoses pubertaires. L'adolescence est en effet un passage induit, imposé par la puberté avec des transformations physiques et des remaniements psychiques majeurs: un ensemble de processus qui visent à l'individuation du jeune adulte, à sa subjectivation, c'est-à-dire à une poursuite-reprise des identifications secondaires, avec de fait un détachement des identifications primaires (ou peut-être un enjolivement, un travail d'ornementation? Car ces identifications primaires servent de fondations aux secondes; elles constituent le terreau sur lequel ces dernières s'édifient).

\section{Conséquences thérapeutiques auprès de l'adolescent: de la zone dangereuse... de transfert}

Les démarches d'évaluation clinique, diagnostique et thérapeutique exigent la prise en compte de l'articulation entre réalité interne et réalité externe. Cela signifie entre autres d'apprécier la qualité d'étayage de l'environnement et la sensibilité du patient à ce dernier (fonction de sa réalité 
interne). L'intentionnalité thérapeutique du soignant (son engagement) est sans doute une réponse à l'appétence objectale de l'adolescent (bien souvent contre-investie par des conduites d'auto-sabotage jusqu'à l'extrême d'un narcissisme négatif destructeur): intensité transférentielle qui demande un dispositif de soins plurifocal et multidisciplinaire.

Le soin serait un art de l'ajustement permanent de la distance relationnelle, travail du lien qui demande une géométrie et une temporalité particulières: clinique de l'instant, du spontané, de l'inouï; clinique de l'agir, clinique du corps en mouvement, qui nécessite un espace dans lequel pourra advenir un temps, une temporalité adulte. Déployer ce corps ému, en émoi, cette puberté qui pousse vers un avant — en avant de soi (l'ex-ister de Henry Maldiney: se tenir hors de soi) — tout en jouant et révélant, en après-coup de l'enfance, ce qui se constitue alors comme l'arrière-soi.

La relation se tisserait de cette trajectoire de soi à l'autre/l'objet, axe narcissico-objectal mis au travail à l'adolescence. Ce lien est vécu comme une menace par l'adolescent quand l'investissement de l'objet attaque l'intégrité narcissique, c'est-à-dire quand il y a un antagonisme entre la relation à l'objet et l'investissement libidinal narcissique. Le lieu de la menace de déséquilibre, de déstabilisation identitaire, est l'espace de confusion entre soi et l'objet, le site de la rencontre, qui est aussi celui du traitement: la zone de transfert.

Quelle est la réponse du dispositif de soin? Il s'agit d'aménager cette relation, pour d'une part la rendre tolérable, et d'autre part permettre le travail de transformation - le traitement psychique. Pour cela, la relation soignant-soigné est tiercéisée par les médiations thérapeutiques, artistiques (Corcos, 2007, 2014) et corporelles, et/ou le groupe de pairs et de soignants (Guedeney et Jeammet, 1991). La charge d'investissement transférentiel (à la mesure du pulsionnel) est diffractée grâce à la pluralité de soignants (Corcos, 1999) : tant en termes de nombre que de qualité (âges différents, sexes, fonctions, formations... et ce qui fait que chacun est unique!); comme si les différentes entités qui composent l'institution portaient et représentaient dans le monde externe une part des motions pulsionnelles de l'adolescent. Cette idée qu'une unité de soin ou un soignant, un groupe, puisse jouer le rôle de support par projection d'une instance psychique, d'une quantité de libido pulsionnelle, ou encore d'une représentation, est à l'origine de la notion d'espace psychique élargi pour penser de façon dynamique la relation entre le patient et l'institution, de même que pour approcher le fonctionnement psychique de l'adolescent. Ainsi, quand le soin est efficient (quand il y 
a engagement de part et d'autre dans le soin), l'institution de chair fait fonction de scène externe disponible pour une mise en représentation du théâtre interne de l'adolescent; l'énergie transférentielle ainsi répartie et divisée en petites quantités peut être traitée plus facilement.

Aussi, jouer de la différence, par petites touches, permet un travail de différenciation à partir du même qui se répète, mais jamais tout à fait identique. Comment penser cette relation de soin singulière, et comment panser (ce lien du transfert qui témoigne de l'atemporalité des premiers soinsliens) ? Il s'agit de figurer avant que de symboliser.

Le soin a fonction première de pare-excitation et de restauration de la continuité de soi, à la fois vecteur et enveloppe. D’où l'importance du vécu, de l'éprouvé, ensemble et pour soi, selon le rythme alterné des battements du temps pulsionnel, entre espaces de confusion et moments de solitude, au fondement de la capacité d'être seul en présence de l'autre (Winnicott, 1971). Proposer, vivre avec, corps à corps, accueillir et contenir, par les gestes, les mots, le groupe. Multisensorialité et transmodal (Daniel Stern) qui nourrissent le travail de mise en concordance et de co-création de liens.

Créer une scène du rêver, l'espace-temps du surgissement de la trouvaille, de l'inédit; une clinique de l'émergence (Daniel Widlöcher), qui est avant tout langage de l'affect (l'affect d'existence), naît de cette tension narcissico-objectale qui suppose à la fois l'écart et le chaos-fusion d'où jaillissent des potentialités de création - voire du trouver-créer — au fondement de l'être, du sentiment d'existence et du sentiment que la vie vaut vraiment la peine d'être vécue.

\section{En conclusion}

Les enjeux de la compréhension de la symptomatologie et de ce fait du traitement peuvent s'envisager selon le triple point de vue métapsychologique de $\mathrm{S}$. Freud:

- la dimension économique (métaphore énergétique) : l'afflux d'excitation avec la puberté (d'origine physiologique et psychique) bouleverse l'équilibre interne, débordant le moi et révélant ses fragilités narcissiques.

- une dynamique paradoxale: les forces pulsionnelles qui attirent l'adolescent vers les figures parentales sont les mêmes qui s'inversent en forces répulsives (sentiment de dégoût et de rejet envers les parents qui traduisent - et en réponse à - l'hypersexualisation des liens). La notion de contre-investissement (dans une acception à la fois 
économique et dynamique), articulée au concept de dépendance, est ici fondamentale pour comprendre la psychopathologie adolescente. Comme le manifeste (le symptôme) masque le latent (fantasme, représentation inconsciente), l'investissement par l'adolescent de la réalité externe, perceptive, trahit la vulnérabilité de son monde interne et une extrême dépendance à l'environnement. Tout lien, potentiellement vecteur d'un état de détresse et d'effroi secondaire à une séparation redoutée et vécue sur un mode anaclitique et subi (passivation intolérable), est de ce fait appréhendé comme particulièrement menaçant. La relation de dépendance est alors maintenue à distance, dans un effort de maîtrise illusoire, par le contre-investissement de la réalité externe (à fonction anti-relationnelle). L'écart (ou l'antagonisme) narcissico-objectal traduit la menace qui pèse sur l'identité du sujet et qui est à la mesure de son besoin pour l'objet quand, selon un double effet d'après-coup à l'adolescence, les assises narcissiques sont ébranlées (révélant des carences infantiles) ou encore que l'environnement s'avère peu étayant narcissiquement. Antagonisme qu'exprime Philippe Jeammet sous forme d'un paradoxe: «ce dont j'ai besoin, parce que j'en ai besoin, et à la mesure même de ce besoin, est ce qui menace mon autonomie» (Jeammet, 1992, p. 3207).

- la dimension topique: la régression à des modalités relationnelles archaïques se traduit par une confusion entre le désir (pour l'objet), l'objet, et le moi (André Green, 1982, p. 195), selon un processus de dé-différenciation qui accroît d'autant la menace provenant de l'objet. Le recours à la réalité externe pallie alors le manque de jeu interne; l'espace perceptif est utilisé comme contre-investissement de l'investissement massif indifférencié et excitant des objets/imagos internes.

Le «cadre» des soins institutionnels, c'est l'importance de l'esprit qui y souffle, qui l'anime, qui nous anime. Cela serait-il de l'ordre du reproductible? Mesurable, prouvé donc exportable (selon la doxa de l'Evidence Based Medecine)? Est-ce que cet esprit repose sur les qualités charismatiques d'une personne unique? Chef fondateur, détenteur d'une autorité qui fédère, puis de sa filiation, ses successeurs qui apportent leur part d' «âme», ou leur pierre? Comment cet édifice peut-il rester vivant, inventif, afin de répondre à la fonction d'aire transitionnelle, aire intermédiaire d'expérience de D. W. Winnicott (Winnicott, 1975), si importante en regard de la clinique adolescente? 
Pour revenir à la notion d'engagement: c'est ce liant, cette énergie émanant du groupe qui tient peut-être d'une mythologie commune, à savoir la croyance ou la conviction intime que le temps d'adolescence serait pour chacun et pour tous un temps bien singulier, particulièrement important. Ainsi nous nous trouverions à la croisée de deux axes «instituant», verticalfiliatif et horizontal-affiliatif:

- la filiation H. Flavigny-P. Jeammet-M. Corcos, qui relève à la fois de la tradition orale et de la transmission écrite;

- le compagnonnage institutionnel, où nous sommes tous institués co-auteurs du contrat institutionnel (J. Hochmann).

Le plaisir à œuvrer ensemble, à penser le matériau d'un vécu commun, écrit une histoire, celle de notre rencontre avec l'adolescent que nous soignons, en résonance avec nos propres adolescences, une histoire qu'il pourra reprendre, poursuivre, nous dire, s'écrire, ré-inventer.

Geste: posture, gestuelle mais aussi épopée, une histoire que l’on (ra)conte en évoquant les temps anciens de la pédopsychiatrie institutionnelle?

Guillemette Balsan

guillemette.balsan@imm.fr

\section{Notes}

1. En référence au slogan de 1968 «il est interdit d'interdire».

2. Numéro d'urgence pour les sans-abri, qui pour les premiers appelants permet de bénéficier d'un hébergement provisoire (pour une nuit).

3. CAC: lieu d'accueil, de soins, d'orientation ou d'hospitalisation de patients en crise; proposant souvent une permanence téléphonique et des consultations psychiatriques d'urgence; le plus souvent sectorisé (répondant à un secteur géographique donné).

4. Association de Santé Mentale du $13^{\mathrm{e}}$ arrondissement de Paris.

5. Traité de psychiatrie de l'enfant et de l'adolescent.

6. Voir Birot et Jeammet (2006).

7. T2A: tarification à l'activité; mode de financement des établissements de santé qui vise à mettre en place un cadre unique de facturation et de paiement des activités hospitalières.

8. RIM-P: Recueil d'Information Médicalisée en Psychiatrie; système qui permet une mesure médicalisée de l'activité hospitalière française ayant une activité en psychiatrie; recueil systématisé d'informations relatives à chaque prise en charge des patients.

\section{Références}

Azoulay, C. et Chabert, C. (1998). Chronique de la vie quotidienne dans un centre de jour pour adolescents: de la théorie à la pratique. Paris: Expansion scientifique.

Bion, W. (1983). Réflexion faite. Paris: Presses universitaires de France.

Birot, E. et Jeammet, P. (2006). Soigner l'anorexie et la boulimie, des psychanalystes à l'hôpital. Paris: Presses universitaires de France. 
Carton, S., Chabert, C. et Corcos M. (2011). Le silence des émotions. Paris: Dunod.

Corcos, M. (1999). La thérapie bifocale dans la dépression de l'adolescent: indications et intérêts. Neuropsychiatrie de l'enfance et de l'adolescence, 47 (4), 191-199.

Corcos, M. (2007). Accueil des adolescents: engagement et créativité. Annales médico-psychologiques, 165 (10), 723-728.

Corcos, M. (2000). Le corps absent approche psychosomatique des troubles des conduites alimentaires. Paris: Dunod, 2010.

Corcos, M. (2005). Le corps insoumis psychopathologie des troubles des conduites alimentaires. Paris: Dunod, 2011.

Corcos, M. (2014). La chambre à soi — apologie des médiations culturelles à l'adolescence. Psychiatrie française, 1, 5-8.

Freud, S. (1923). Le moi et le ça. Dans Essais de psychanalyse, Paris: Payot, 1968.

Green, A. (1982). Après coup l'archaïque. Nouvelle Revue de psychanalyse, 26, 195.

Guedeney, N. et Jeammet, P. (1991). Objectifs et diversité des modalités du travail des équipes soignantes avec les parents d'adolescents hospitalisés. Neuropsychiatrie de l'enfance et de l'adolescence, 39, 533-539.

Hochmann, J. (1994). La consolation: essai sur le soin psychique. Paris: Odile Jacob.

Jeammet, P. (1980). Réalité externe, réalité interne. Importance et spécificité de leur articulation à l'adolescence. Revue française de psychanalyse, 44 (3-4), 481-521.

Jeammet, P. (1989). Psychopathologie des troubles des conduites alimentaires à l'adolescence. Valeur heuristique du concept de dépendance. Confrontations psychiatriques, 31, 177-202.

Jeammet, P. (1992). Pathologie de la dépendance à l'adolescence. Médecine et hygiène, 50, 3206-3208.

Jeammet, P. (1996). Psychanalyse et psychiatrie: des relations toujours actuelles. Revue française de psychanalyse, 60 (2), 351-367.

Jeammet, P. (2002). Spécificités de la psychothérapie psychanalytique à l'adolescence. Psychothérapies, 22, 77-87.

Racamier, P.C. (2008). Le psychanalyste sans divan: la psychanalyse et les institutions de soins psychiatriques. Paris: Payot.

Tric L., Agman G., Godart N., Benmansour E.L., et Lamer C. (2004). Prise en charge de l'anorexie mentale en réanimation. Réanimation, 13 (6-7), 407-416.

Winnicott, D. W. (1954). La nature humaine. Paris: Gallimard, 1990.

Winnicott, D. W. (1971). Jeu et réalité. Paris: Gallimard, 1975. 